\title{
Regen, Erdbeben und Klimawandel. Die Katastrophe der unrechtmäßigen Herrschaft in antiker literarischer Tradition
}

\author{
Sabine Müller
}

\begin{abstract}
This paper explores the political interpretation of natural disasters in ancient literary tradition. Regarded as divine signs or punishments, a natural phenomenon or disaster is often connected to illegitimate rule. Specifically, the paper will examine the connection between natural disasters and political changes in ancient literature. It will be argued that descriptions of natural disasters often serve either to emphasize or to hide violations of political tradition or continuity of power.
\end{abstract}

Keywords: natural disasters; legitimacy; continuity; tradition; political systems

\section{Naturkatastrophen im antiken Herrschaftsdiskurs}

Antike Naturkatastrophen sind seit einigen Jahren verstärkt in den Blickpunkt des Interesses der althistorischen Forschung gerückt. ${ }^{1}$ Neben der Frage nach den Formen der Bewältigung, dem Krisenmanagement und der Vorbeugung von Katastrophen steht vor allem der antike Wahrnehmungs- und Deutungshorizont im Vordergrund der Untersuchungen (Sonnabend 1995). Als dominierend ist die straftheologische Auslegung von Naturkatastrophen im Sinne eines deutlichen Fingerzeigs von göttlicher Seite zu betrachten: als - mehrheitlich schlechte - Vorzeichen, Warnungen oder Bestrafungen (Groh/Kempe/Mauelshagen 2003, 20; Sonnabend 2003, 43f.; Kehne 1998, 374; Mylonopoulos 1998, 82f.). ${ }^{2}$ Aus diesem vorherrschenden Deutungsmuster resultierte die politische Instrumentalisierbarkeit von Naturkatastrophen (Waldherr 1998, 63; Sonnabend 1998, 34). Es verwundert daher nicht, dass Beschreibungen von Umschwüngen und verstörenden Phänomenen in der Natur als „Herausforderung für Herrschaft und ihre Legitimation“ (Groh/Kempe/Mauelshagen 2003, 26) zu den gängigen Motiven der Darstellung

1 Zur Definition des Begriffs „Naturkatastrophe“ vgl. Groh/Kempe/Mauelshagen 2003, 15-17.

2 Dieser Deutungshorizont entspricht insbesondere dem griechischen pessimistischen Bild der olympischen Götter, die mit Neid und Eifersucht auf die Menschen sehen und sie zu Fehlern verleiten, um sie dann dafür zu bestrafen, ein zentrales Thema etwa der attischen Tragödie. Vgl. Heinrichs 1989, 97; Zimmermann 1986, 38, 113; Marcuse 1985, 33-34. 
unrechtmäßiger Regentschaften in der antiken literarischen Tradition gehören. Nicht zwangsläufig reflektieren sie historische Ereignisse, sondern können auch fiktiv sein, um ein Argument zu unterstreichen (Sonnabend 1998, 35f.). Das Aufbäumen der Natur, häufig im Modell der Inversion ausgedrückt, vermittelt, dass es sich um einen Ausnahmezustand handelt, der als Gegenbild des einst in mythischer Vorzeit von den Göttern etablierten Idealzustands imaginiert wird. Um einem antiken Rezipientenkreis zu suggerieren, dass eine politische Ordnung unrechtmäßig war, erschienen daher Motive einer aus den Fugen geratenen Natur als instruktiv.

Die folgende Untersuchung wirft einen differenzierteren Blick auf die Deutung des Zusammenhangs zwischen Naturkatastrophen und Herrschaft in der antiken literarischen Tradition. Anhand von vier exemplarischen Fällen aus dem Bereich des antiken Ostens sowie dem frühen Hellenismus wird dargelegt, dass Elemente der Katastrophentopik in politischen Diskursen auffallend häufig im speziellen Kontext von Brüchen der Herrschaftskontinuität und -tradition auftauchen. Bei Konstellationen des politischen Wandels und der Verlagerung von Entscheidungsbefugnissen in ihren verschiedenen Formen Usurpationen, Fremdherrschaften, Dynastiewechseln oder dem Erlöschen von Herrscherhäusern - sind Naturphänomene ein fast gesetzmäßiges Begleitmotiv. ${ }^{3}$ Daraus ist zu schließen, dass die Katastrophentopik gezielt instrumentalisiert wurde, um die in einer Krisensituation entstandenen Traditionsbrüche, abhängig vom Darstellungsinteresse, entweder zu neutralisieren oder besonders hervorzuheben und das entstandene Bild im kulturellen Gedächtnis zu verankern. Als zentrale Referenz und bedeutender Legitimationsfaktor wurde die politische Tradition mit Hilfe der Katastrophenszenarien neu interpretiert, definiert oder konstruiert. Teils erfuhr sie auch eine chronologische Verlängerung bis zurück in mythische Urzeiten.

Im Rahmen der Untersuchung wird zudem aufgezeigt, dass zwischen den verschiedenen Rollen der Götter in den antiken Deutungsmustern von Naturkatastrophen und phänomenen zu differenzieren ist. Nicht in allen Fällen ist von einem göttlichen „Denkzettel“ für menschliches Fehlverhalten auszugehen, unter dem auch der unrechtmäßige Herrscher selbst zu leiden hat. Daneben existiert die Variante, nach welcher dieser bewusst dafür sorgt, dass sich die göttlichen Vorgaben umkehren, indem er die Tempel zerstört und die Götter als überirdische Garanten der Ordnung und Wächter über die Naturverhältnisse vertreibt. Er selbst fühlt sich im ausbrechenden Chaos als seinem eigenen Element wohl.

\section{Naturkatastrophen als Vorboten und Begleiterscheinungen von Fremdherrschaft}

Der Wechsel von einer als legitim zu einer als illegitim konnotierten Herrschaft wird in der antiken literarischen Tradition häufig von außergewöhnlichen Naturereignissen in der Funktion eines schlechten Omens angekündigt. Ein instruktives Beispiel bietet der Bericht des griechischen Historiographen Herodot aus dem 5. Jh. v. Chr. über die Eroberung Ägyptens 525 v. Chr. unter dem persischen Großkönig Kambyses (530-522 v.

3 Diesbezüglich erscheint es interessant, dass die moderne Katastrophenforschung von einem Zusammenhang zwischen Katastrophenhäufigkeit und Gesellschaften im soziokulturellen Umbruch ausgeht (Waldherr 1998, 62). 
Chr.). Der aus der kleinasiatischen Griechenstadt Halikarnassos stammende Herodot hatte auf seinen Reisen vor Ort in Theben, Memphis und Heliopolis recherchiert und einheimische Priester zur persischen Eroberung Ägyptens befragt, die er im Zuge seiner Darlegung des Aufstiegs Persiens thematisierte (Hdt. 2,3,1). ${ }^{4}$ Was sie ihm vermittelt hatten, war eine Negativversion der Ereignisse, die, wie John Dillery aktuell darlegte, nach dem Modell der ägyptischen Unheilsschilderungen geformt und mit den charakteristischen Stilelementen versehen war (Dillery 2005, 387-406). In Folge figuriert Kambyses in Herodots Historien als ein grausamer, von Wahnsinn geschlagener Tyrann, der die ägyptische Religion verspottete, Heiligtümer und Gräber entweihte und als Krönung den heiligen Apisstier in Memphis tötete (Hdt. 3,16, 28f.. 37. Vgl. Desmond 2004, 29; Briant 1996, 69f.; Bresciani 1996, 503f.; Munson 1991; Balcer 1987, 73; Brown 1982). Der Frevel gegen den Tierkult gilt als eine Schlüsselszene seiner Negativporträtierung: „Als nun die Priester den Apis brachten, zog Kambyses, halb von Sinnen wie er war, seinen Dolch und wollte ihn dem Apis in den Bauch stechen, trifft aber den Schenkel. Da lachte er und sprach zu den Priestern: ,Ihr Gauner, sind Götter wohl von der Art, dass sie Blut und Fleisch haben und das Eisen spüren?"“ (Hdt. 3,29, 1f.). ${ }^{5}$ Dieser Tendenz entspricht auch die Schilderung von Kambyses' vorangegangener Ankunft in Ägypten, die Herodot von den Priestern übernahm. Demnach kündigte sich sein Eintreffen mit einem einmaligen Naturereignis an: „Es fiel nämlich Regen im ägyptischen Theben, wo es weder vorher jemals geregnet hat noch nachher bis auf meine Zeit, wie die von Theben selbst sagen. Es fällt nämlich in Oberägypten überhaupt kein Regen, damals aber fiel er in Theben, ein richtiger Nieselregen“ (Hdt. 3,10, 3). ${ }^{6}$ Entgegen der These, dass Kambyses somit als Heilsbringer dargestellt worden sei, der dem Land fruchtbaren Regen gebracht habe (Huß 1997, 132), weist die Gesamtausrichtung seines Porträts bei Herodot vielmehr darauf hin, dass es sich bei dem geschilderten Phänomen um ein Element der ägyptischen Unheilsschilderungen handelt (vgl. Harrison 2003, 154). Der ungewöhnliche Regen war ein Symbol, mit dem Kambyses' Eintreffen zur Verheißung von Unglück stilisiert wurde. Wenn es, wie die Tradition impliziert, in der alten Königsstadt Theben unter den legiti-

4 Herodot beschrieb in seinen Historien die Vorgeschichte (den Aufstieg Persiens) und Ereignisse der Perserkriege. Der Zusammenprall zwischen Persern und Griechen führte dazu, dass das Perserreich in griechischer Tradition häufig stereotyp als dekadente Tyrannei geschildert wurde. Vgl. Briant 2002; Lateiner 1989, 154; Raaflaub 1985, 72-81. Herodot betrachtet fremde Völker wie auch die Perser jedoch nicht aus der in seiner Zeit gängigen tendenziösen Perspektive (Heinrichs 2001, 1169f.; ders. 1989, 129154; Müller 2007, 17f.). Dass die Großkönige Kambyses und Xerxes bei ihm gemäß Tyrannentopik porträtiert sind (Lateiner 1977, 177f.), ist ein komplexes Phänomen. Im Fall von Kambyses, der als ein Herrscher figuriert, der sowohl fremde als auch persische Sitten missachtet (Hdt. 3,16,3. 38,1. Vgl. Desmond 2004, 19-40; Fornara 1990, 41, 45; Lateiner 1989, 145-155; Evans 1965, 147-153) war wohl die ägyptische Negativtradition prägend. Tuplin (1991, 268f.) und Balcer $(1987,91-93)$ vermuten zudem, dass die Propaganda Dareios' I. das Bild von Kambyses in Ägypten schwärzte. Von Xerxes, der Griechenland angegriffen hatte, konnte Herodot, der den letzten Teil seiner Historien in Athen verfasste, wohl im Interesse der politischen Kreise, in denen er verkehrte (Heinrichs 1989, 154), vermutlich kein neutrales Bild gestalten. Vgl. Sancisi-Weerdenburg 2005; Gammie 1986, 183-185. Zu Herodots Umgang mit seinen Quellen allgemein vgl. Murray 2001. Speziell zu Herodots ägyptischen Quellen vgl. Stephens 2003, 44-49; Harrison 2003, 145-155; Lloyd 2002, 419; ders. 1990, 224; Kuhrt 1995, II, 664; Munson 1991, 47-54.

5 Herodot-Passagen werden zitiert nach der Übersetzung von W. Marg.

6 Wahrscheinlich kannte Herodot das zugrunde liegende literarische Modell der ägyptischen Unheilsschilderungen jedoch nicht (zur Diskussion siehe Anm. 4) und deutete die Geschichte vom Regen in Theben, die ihm erzählt wurde, daher nicht per se als negativ. 
men ägyptischen Pharaonen - den Abbildern des Horus auf dem Thron (Clauss 2001, 69; Turner $1984^{2}, 132$ ) - nicht geregnet hatte, konnte dies nur bedeuten, dass ein trockenes Klima in diesem Gebiet dem Idealzustand der göttlichen Ordnung entsprach. Kehrten sich aber die klimatischen Bedingungen gerade zu dem Zeitpunkt komplett um, als Kambyses sich der Küste näherte, verwies das Phänomen auf den Anbruch einer Unheilszeit unter einem unrechtmäßigen Herrscher, der gegen die göttlichen Vorgaben verstieß. Der Regen über Theben ist als ein symbolischer Code zu sehen; ob er ein fiktiver Zusatz oder historisch war und den Priestern in ihrer Deutung der Ereignisse gerade recht kam, ist ungewiss. ${ }^{7}$ In jedem Fall warnte das schlechte Omen des Niederschlags vor Kambyses' kommenden Freveln gegen die religiösen und politischen Vorgaben Ägyptens.

$\mathrm{Zu}$ dieser Negativdarstellung des Großkönigs stehen ägyptische Parallelquellen im Widerspruch, die davon zeugen, dass Kambyses die einheimische Religion respektierte und sich in die politische Tradition der Pharaonen stellte, um seine Herrschaft in dem neu eroberten Land zu konsolidieren (Dillery 2005, 400f.; Vittmann 2003, 126; Baines 1998, 86-93; Cook 199632, 214). So ließ er sich eine pharaonische Titulatur erstellen, stiftete einen teuren Sarkophag für den verstorbenen Apisstier, wohnte den Trauerzeremonien bei und setzte einen neuen Stier ein (Dillery 2005, 400f.; Briant 1996, 67f.; Kuhrt 1995, II, 663f.; Posener 1936, 33-36). Diese Dokumente für den respektvollen Umgang mit der ägyptischen Kultur - wenn sie auch aus politischem Kalkül erfolgte - machen es unwahrscheinlich, dass Kambyses als Apismörder und Tempelschänder in Ägypten auftrat (Serrano Delgado 2004, 34; Briant 1996, 70, 109). ${ }^{8}$ Vielmehr handelt es sich um topische Elemente der ägyptischen Chaosbeschreibungen, in denen es zu den Charakteristika des unrechtmäßigen Herrschers gehört, gegen den Götterkult zu freveln (Dillery 2005, 396). Kambyses wurde zum Verhängnis, dass der Apisstier während seines Aufenthalts in Ägyptens starb; in der Rezeption durch perserfeindliche Priesterschaften wandelte sich dieses Ereignis zur Ermordung des Tiers (Dillery 2005, 396; Bresciani 1996, 504f.). Die Ressentiments von Teilen der ägyptischen Priesterschaft gegen Kambyses, die sich in seiner Verfemung ausdrückten, resultierten neben seiner mangelnden Tempelbautätigkeit vor allem aus seinem Erlass zur Reform der Finanzen der Tempel, die massive Kürzungen ihrer Einnahmen bedeutete und sie empfindlich traf (Serrano Delgado 2004, 42; Vittmann 2003, 128f.; Cook 1996², 214; Briant 1996, 71).

Das Beispiel des Regens in Theben zeigt, wie ein Bruch mit der Herrschaftskontinuität - in diesem Fall aufgrund einer Eroberung - von einheimischen, wenig kooperationsbereiten Kreisen mittels der Schilderung eines Naturphänomens als ein literarisches Stilmittel betont wurde. Im kulturellen Gedächtnis Ägyptens ging die Epoche der persischen Herrschaft symbolisch codiert als Unheilszeit ein (vgl. Curt. 4,7,1). Die positiven Berichte zu Kambyses verblassten, das Zerrbild des grausamen Tyrannen, der mit seinen verbrecherischen Horden das Land unterjocht hatte, dominierte (Clauss 2001, 446).

Dies zeigen die Fragmente ägyptischer prophetischer Schriften als zweites Beispiel. Sie entstanden während der Zeit der persischen Kontrolle in Priesterkreisen, setzten sich mit der Situation auseinander und wurden während der späteren makedonischen Ptolemä-

7 Es mag zudem eine Rolle gespielt haben, dass die Entscheidungsschlacht der Perser gegen die Ägypter eine Seeschlacht (bei Pelusion) gewesen war (Hdt. 3,10,1).

8 Dagegen argumentieren aktuell für eine Ermordung des Apis durch Kambyses: Vittmann 2003, 126; Depuydt 1995, $201 \mathrm{f}$. 
erherrschaft aktualisiert (Bresciani 1998, 280; Huß 1994). Teils richteten sie sich gegen die makedonischen Fremdherrscher, teils, wenn die Tempel mit dem ptolemäischen Regime kooperierten, gegen deren politische Gegner, die Seleukiden in Syrien. Da die Seleukiden Teile des ehemaligen Perserreichs beherrschten, figurierten sie als Nachfolger der Großkönige und damit als Vertreter des Chaos (Thissen 2002, 123-136; Moyer 2002, 80; Hoffmann 2000, 184; Funck 1996; Hölbl 1994, 73f.).

Die prophetischen Schriften haben die Eroberung Ägyptens durch auswärtige Invasoren und ihre Folgen zum Thema. Die Fremdherrschaft erscheint als eine Phase des Chaos, die das Land zu einer verkehrten Welt gestaltet. Die soziale Ordnung und die Natur stehen auf dem Kopf; es regieren Mord und Totschlag. Der Götterkult wird vernachlässigt oder aufgehoben, als Konsequenz verlassen die Götter das Land. Das Land wird unfruchtbar und die Jahreszeiten spielen verrückt, wie im Orakel des Töpfers beschrieben wird: „Die Flut (wird nicht mit genug) Wasser (kommen), sondern mit nur wenigem, so dass (die Erde) in Brand gerät (...). (Die Sonne) wird blass werden, weil sie das Übel in Ägypten nicht sehen will. Die Erde wird auf die Samenkörner nicht ansprechen. Ihre meisten werden vom Sturm zerstört sein" $\left(\mathrm{P}_{2}\right.$ 1-8). ${ }^{9}$ Die Folgen sind Hungersnöte, Verarmung und Bürgerkriege (Blasius/Schipper 2002, 285f.). Die Perser werden als eine räuberische Bande geschildert, die wie ein Heuschreckenschwarm über Ägypten einfällt, Tempel plündert, in der Bevölkerung Blutbäder anrichtet und die Überlebenden in eine bedrückende Knechtschaft zwingt: „Unsere Seen und unsere Inseln sind voll von Tränen. D.h.: Die Häuser der Ägypter, sie werden keine Menschen mehr haben, die in ihnen wohnen (...) Die Meder werden sie niedermetzeln, [sie] werden ihre Häuser nehmen und in ihnen wohnen" (Demotische Chronik IV/21). ${ }^{10}$

Im Lamm des Bokchoris wird der Anbruch der persischen Terrorherrschaft des Chaos, Unheils, der Ungerechtigkeit und Inversion durch ein sprechendes Lamm prophezeit: „Weh, Ägypten, (das weint) wegen der Verfluchung, die zahlreich auf ihm lastet! (...) man wird die Straßen von Sebennytos zu einem Weingarten machen, während der Teich von Mendes ein Gebüsch von Gurken und Kürbissen ist (...) es weint Memphis (...), es weint Theben (...) auf Furcht folgt Leiden.“" ${ }^{\text {"I }}$ (I/14-18. Vgl. Hoffmann 2000, 182). Diese Leidenszeit wird beendet, wenn ein ägyptischer Heilskönig auf den Plan tritt und mit seiner Inthronisation die göttliche Ordnung wieder etabliert (Blasius/Schipper 2002, 283286; Hoffmann 2000, 177). „Aber was den Meder betrifft, der sich Ägypten zugewendet hat - er wird sich zu den Fremdländern entfernen. Die Wahrheit wird ans Licht kommen, die Lüge wird zugrunde gehen. Recht und Ordnung werden in Ägypten herrschen“ (Lamm II/21-23). Der Lauf der Natur reguliert sich wieder: „Am Ende der Übel wird (...) der wasserarme Nil (...) voll werden; der Winter, der in unpassender Weise sich seines Gewandes entkleidet hatte, wird in seinen eigenen Kreislauf kommen, und dann wird der Sommer seinen eigenen Lauf nehmen. Das Wehen der Winde, welches (früher zerstörerisch war (?)) wird milde abgeschwächt und wohl geordnet sein“ (Töpfer $\mathrm{P}_{3} 43 \mathrm{ff}$.).

Die prophetischen Texte leiten sich vom Mythos um Osiris, Seth und Horus ab, dem auch die ägyptische Geschichtsauffassung und -deutung zugrunde liegt (Hoffmann 2000, 176; Wildung 1977, 565). Der archetypischen Darstellung zufolge regierte in der mythi-

9 Passagen aus dem Orakel des Töpfers werden zitiert nach der Übersetzung von L. Koenen.

10 Zitiert nach der Übersetzung von H. Felber.

11 Passagen aus Lamm des Bokchoris werden zitiert nach der Übersetzung von H.-J. Thissen. 
schen Vorzeit Osiris als göttlicher Idealkönig und Zivilisator auf Erden. Sein neidischer Bruder Seth, negativer Gegenpol und Verkörperung des Chaos, ermordete ihn und löste mit seiner Usurpation eine Zeit des Unheils aus. Osiris' Schwestergemahlin Isis begab sich auf die Suche nach seinem Leichnam und empfing postum ihren Sohn Horus von ihm, Prinzip des Guten und Gerechten. Vor Seths Nachstellungen geschützt, wuchs Horus versteckt in den Sümpfen des Nildeltas auf und wurde von seinem Vater Osiris aus dem Totenreich für seinen Kampf gegen Seth trainiert. Am Ende siegte das Prinzip des Guten, Horus, über das Chaos (Plut. mor. 351 C-384 C; Diod. 1,21,2-22,7). Mit Horus' Thronbesteigung wurde die göttliche Ordnung wieder hergestellt und die Leidenszeit für Ägypten respektive für die Welt - da der ägyptische König der Theorie nach Universalherrscher war - beendet. Dieser Mythos lag auch der herrschaftlichen Ideologie zugrunde, nach der jeder Pharao das Bild des Horus aus dem irdischen Thron darstellte und das Chaos, das kurzzeitig bei jeder Vakanz Oberhand gewann, mit seiner Inthronisation beendete (Clauss 2001, 69; Hoffmann 2000, 176; Assmann 1991, 127). ${ }^{12}$

Die prophetischen Schriften spiegeln in ihren Versionen, die sich gegen die persische Herrschaft richten, die Hoffnung auf eine Rückkehr zur pharaonischen Tradition wider. Die Verfasser verlagerten die Niederlage gegen die Eroberer auf eine transzendente Ebene und stellten einen Wandel der politischen Verhältnisse in Aussicht, der sich aus dem Kreislaufschema der ägyptischen Geschichtsauffassung ergab. In der Aktualisierung der Texte in ptolemäischer Zeit wird der Bruch der Herrschaftskontinuität entweder unterstrichen oder, indem die Makedonen als die rettenden Heilskönige porträtiert werden, die nach den Persern kamen, vertuscht. Bezüglich der Motive der Verfasser ist zu folgern, dass sie sich entweder von den Fremdherrschern distanzieren oder ihre kooperationsbereite Haltung rechtfertigen wollten.

Blickt man auf die Seite der Fremdherrscher, musste den Ptolemäern daran gelegen sein, in den Augen der einheimischen Bevölkerung als Gegenbilder zu den übel beleumundeten Persern zu gelten. Da Alexander der Große bei seiner Eroberung Ägyptens 332/1 v. Chr. die Perser abgelöst hatte (Arr. an. 3,1,1-2; Curt. 4,7,1-2; Diod. 17,49,2) und die Ptolemäer seine Nachfolger in Ägypten waren, konnten sie für sich in Anspruch nehmen, eben die ersehnten Heilskönige zu sein, die das Chaos beendet hatten.

\section{Naturkatastrophen als Motiv von Legenden der Herrschaftsgründung}

Das dritte Beispiel illustriert, wie Schilderungen von Unheilsherrschaft als Teil einer Legitimationsstrategie eingesetzt wurden, um nachträglich den Bruch von Herrschaftskontinuität zu kaschieren: Thematisiert wird, wie Dareios I. (522/21-486 v. Chr.), der eigentliche Gestalter des Perserreichs, seinen Herrschaftsantritt rechtfertigte.

Nach seiner Machtübernahme, die mit der bisherigen politischen Tradition seit der Reichsgründung unter Kyros dem Großen gebrochen hatte, trieb er großen Aufwand, um

12 In den Pyramidentexten heißt es: „Darauf kam es zwischen dem herangewachsenen Hor und Seth zum Kampf (...) doch schließlich siegte Hor (...) Als dann Geb die Götter im Fürstenhause zu Heliopolis zum Gericht versammelt hatte, da leugnete Seth vergebens (...) Da beugte Hor den Seth unter Osiris (...) Osiris nahm sich jede Krone und Geb setzte ihn auf seinen Thron. Da herrschte er nun als Gott, der keinen Feind mehr hat“. Zitiert nach Hopfner 1967, 16f. Vgl. Assmann 2004, 16. 
seine Version der Ereignisse publik zu machen. Er ließ einen inschriftlichen Bericht und ein illustrierendes Relief (Cool Root 1979, 189) an der Felswand von Behistun anbringen, einem Bergmassiv, das an einer der Hauptkarawanenstraßen zwischen dem mesopotamischen Tiefland um Babylon und dem iranischen Hochland gelegen war (Wiesehöfer 20053 , 33). Zudem wurden Kopien durch das ganze Reich verschickt (DB IV § 70). In diesem offiziellen Bericht schildert Dareios die Vorgeschichte zu seiner Thronbesteigung folgendermaßen: Während König Kambyses auf seinem Ägyptenfeldzug war, gab sich der betrügerische Hochstapler Gaumata als Kambyses' Bruder Bardiya aus und usurpierte den Thron. Der echte Bardiya war zu dem Zeitpunkt schon tot, was nicht allgemein bekannt war, da Kambyses ihn aus Argwohn vor seinem Aufbruch nach Ägypten heimlich hatte beseitigen lassen. Als Kambyses auf dem Rückweg nach Persien starb, war niemand im Reich in der Lage, den Betrüger auf dem Thron zu entlarven und ihm das Handwerk zu legen. Er brachte Unheil über das Land, bis er von Dareios aufgehalten wurde. Zusammen mit sechs Vertretern der einflussreichsten persischen Familien gelang es ihm, Gaumata zu stürzen (DB I § 10-15). Danach übernahm er die Herrschaft und schlug in einem Jahr die gegen ihn ausbrechenden Widerstände im Reich nieder (Kuhrt 1990, II, 665): „Diese Länder, die abtrünnig wurden, die Lüge hat sie abtrünnig gemacht, so dass diese (Männer) das Volk belogen. Daraufhin hat Auramazdah sie in meine Hände gegeben“ (DB IV § 54). ${ }^{13}$ Dareios schildert die von ihm gestürzte Regierung als Bruch mit der Herrschaftstradition, seine Thronbesteigung dagegen als die rettende Heilsherrschaft nach dem Chaos. Darüber hinaus berief er sich darauf, an eine weitaus ältere, noch vor Kyros' Regierung anzusiedelnde Herrschaftstradition anzuknüpfen.

In der aktuellen Forschung dominiert die Position, dass Dareios eine so nachdrückliche Legitimation nötig hatte (Rollinger 2006, 48; Wiesehöfer 2005³, 63), da er nur einem Nebenzweig von Kyros' Dynastie entstammte (Briant 1996, 122-124; Heinrichs 1987, 504f.; Balcer 1984, 158-160), woran teils auch Zweifel bestehen (Brosius 1998, 58f.), und vermutlich, wie die Revolten gegen ihn zu zeigen scheinen, keinen betrügerischen Prätendenten, sondern den echten Kyrossohn und legitimen Herrscher Bardiya gestürzt hatte, um an die Macht zu kommen (Brosius 1998, 57; Demandt 1996, 11-20; Cook 19963 ${ }^{3}$, 216; Kuhrt 1995, II, 664f.). ${ }^{14}$ Vor diesem Hintergrund erscheint es erklärlich, dass Dareios auf das altorientalische Schema der Unheilsbeschreibungen zurückgriff. Seine Beschreibung von Gaumatas Regierung enthält deutliche Parallelen zu den ägyptischen Chaosbeschreibungen (Müller 2008): „Vieles wurde schlecht getan, was ich wieder gut machte. Die Länder waren in Aufruhr, ein Mann brachte den anderen um. Nach dem Willen Auramazdahs brachte ich zustande, dass der eine den anderen nicht tötet und dass alles an seinem rechten Platz ist" (DSe $\S 4,30-41) .{ }^{15}$ Gaumata verhält sich spiegelverkehrt zum Idealbild des persischen Königs, der die Bevölkerung ernähren und beschützen, den Götterkult sorgfältig pflegen und das Land sichern, ausbauen und erblühen lassen soll (Wiesehöfer 20053, 59 ). ${ }^{16}$ Dafür bringt die Reichsbevölkerung dem König gemäß der Ideologie der pax persica Respekt entgegen und schätzt sich glücklich, unter ihm zu leben. Gaumata hingegen vernachlässigt und frevelt gegen den Götterkult, beraubt und

13 Passagen aus DB werden zitiert nach der Übersetzung von R. Borger und W. Hinz.

$14 \mathrm{Zu}$ einem Überblick über die verschiedenen Positionen zur Identität des Gaumata vgl. Rollinger 2006, 5153.

15 Passagen aus DSe, DPd und DNa werden zitiert nach der Übersetzung von R.G. Kent.

16 „Über dieses Land möge kein feindliches Heer, keine Hungersnot, keine Lüge kommen“ (DPd § 3). 
unterjocht die Bevölkerung, fördert Gewalt und löst Furcht und Unsicherheit aus: „Das Volk hatte gewaltig vor ihm Angst, weil er viele Leute umbrachte (...) Ich errichtete die Heiligtümer wieder, die Gaumata der Magier zerstört hatte. Ich gab dem Volk zurück die Gehöfte, das Vieh und das Gesinde (...), die Gaumata (...) ihnen geraubt hatte. Ich setzte das Volk wieder an seinen Platz (...) So wie (es) vorher (gewesen war), schaffte ich wieder herbei, was weggenommen worden war (...) Deswegen stand Auramazdah mir bei, sowie die anderen Götter (...), weil ich nicht treulos war, kein Lügenknecht, kein Gewalttäter (...) Nach Gerechtigkeit bin ich verfahren. Weder einem Schwachen noch einem Mächtigen habe ich Gewalt angetan“ (DB I § 13-14; IV § 63).

Das Ausscheren der Natur wird in der Behistun-Inschrift nicht explizit behandelt wie in den ägyptischen prophetischen Schriften, drückt sich aber darin aus, dass die rechte Ordnung gestört war: „die Lüge nahm im Land überhand, in Persien, in Medien und in den sonstigen Ländern“ (DB I § 10).

Da die Götter nicht auf Gaumatas Seite standen, konnte der regelmäßige Lauf der Natur nicht mehr gewährleistet sein. Wie es anders ging, zeigt Dareios auf: „Wer Auramazdah verehrt, der ist auf (immer der rechten Ordnung) teilhaftig“ (DB I § 73).

Dieser Vorstellung entspricht, dass ein Sinnbild des östlichen Herrschers das Amt des Gartenbauers war (Demotische Chronik V/18). Dies kommt etwa in der Akkadischen Sargonlegende, dem Aufstiegsmythos des Urmodells des östlichen Weltherrschers, Sargon von Akkad, zum Tragen, der erst Gartenbau lernte, bevor er von der Göttin Ištar zum König berufen wurde (Kuhrt 2003; dies. 1995, I, 48f.; Binder 1964, 162f.): „Ich bin Sargon, der mächtige König, König von Akkad. (...) Meine Mutter (...) empfing mich, im Verborgenen gebar sie mich. Sie legte mich in einen Korb aus Schilf (...) Sie überließ mich dem Fluss (...) Der Fluss (...) brachte mich zu Aqqi, dem Wasserschöpfer (...) Aqqi zog mich als seinen adoptierten Sohn auf (...), machte mich zu seinem Gärtner. Während ich Gärtner war, gewann Ištar mich lieb, für (...) Jahre übte ich die Königsherrschaft aus. “17 Da der Herrscher auserwählt war, für die irdische Einhaltung der göttlichen Ordnung einzutreten, konnte das Land unter dieser Maßgabe im Wechsel der Jahreszeiten erblühen. Stellte er sich gegen die Götter, kehrte sich die Ordnung und mit ihr die Natur um.

Der Vorstellung vom Sieg des Heilskönigs über das Chaos entspricht auch Dareios' Bericht, dass der höchste persische Gott Auramazdah nur ihn allein als befähigt dafür erkannte, den Usurpator zu stürzen: „Da war niemand, weder ein Perser noch ein Meder (...), der jenem Gaumata (...) die Königsherrschaft entrissen hätte (...) Keiner wagte, etwas auszusagen über (...) Gaumata, bis ich kam“ (DB I § 13).

Dareios' Legitimation gründete sich auf drei Faktoren: göttlicher Berufung, eigener Leistung und königlicher Herkunft, die er in der Behistun-Inschrift wiederholt anspricht. Er betont, seiner Familie ihre einstige Position zurückgegeben zu haben: „Die Königsherrschaft, die unserem Geschlecht entrissen worden war, holte ich zurück und setzte sie an ihren Platz so wie früher" (DB I § 14). Zudem nennt er konkrete Zahlen: „seit alters war unser Geschlecht königlich (...) Acht meines Geschlechts waren vordem Könige. Ich bin der neunte.“ (DB I § 3-4). Die Historizität dieser Herrschaftstradition ist in der Forschung umstritten. Sie führt in die Zeit vor der Gründung des persischen Reichs unter Kyros zurück und bezieht sich wohl auf die Stellung von Dareios' Familie, den Achaime-

17 Zitiert nach der Übersetzung von B. Studevent-Hickman und C. Morgan. 
niden, als Lokaldynasten von Anšan. Dabei handelt es sich um das Gebiet um Pasargadai und Persepolis (nordwestlich des heutigen Shiraz), von dem aus Kyros seinen Eroberungszug gestartet hatte. ${ }^{18}$ Es ist davon auszugehen, dass Kyros' Linie, die Teispiden, Dareios' Familie zu diesem Zeitpunkt in ihrer führenden Stellung in Anšan verdrängt hatte (Kent 1946, 208-211).

Entgegen der vorherrschenden Forschungsposition gibt es keinen Grund, an Dareios' Aussage zu zweifeln, dass vor ihm acht seiner Ahnen in Anšan geherrscht hatten. In einer face-to-face-society wie der persischen Adelsgesellschaft konnte Dareios es sich nicht leisten, seine Thronansprüche auf eine erschwindelte Genealogie zu bauen. Vielmehr war es wohl gerade seine herrschaftliche Ahnenreihe, die es ihm ermöglichte, seinen Thronanspruch gegenüber der persischen Konkurrenz durchzusetzen. Die Ikonographie von Dareios' Darstellung auf dem Behistun-Relief scheint zu untermauern, dass er sich auf die einstige Führungsposition seiner Familie in Anšan bezog: Er trägt das Herrschaftssignum des obersten Dynasten von Anšan, das Diadem (Nagel 1972/75, 358).

Was Dareios in seinem Rechtfertigungsbericht unternimmt, ist eine Rekonstruktion von persischer Herrschaftstradition, zu deren Bestandteilen auch das Katastrophenszenario gehört. Seiner Aussage zufolge trat der Kontinuitätsbruch nicht mit seinem Regierungsbeginn ein, sondern erfolgte mit der Thronbesteigung seines Amtsvorgängers Gaumata-Bardiya, die eine Unheilszeit auslöste. Dareios' Herrschaftsantritt kittete hingegen diesen Bruch - „Auramazdah, als er diese Erde in Aufruhr sah, übertrug sie daraufhin mir, verlieh mir die Königsherrschaft. Nach dem Willen Auramazdahs setzte ich sie an ihren Platz zurück" (DNa § 4) - und bedeutete die Anknüpfung an eine politische Tradition von beeindruckender Dauer. In dieser Rekonstruktion von Vergangenheit gelingt es Dareios gleich zwei Brüche von Herrschaftskontinuität zu neutralisieren: den Machtwechsel von den Achaimeniden zu den Teispiden in Anšan und den Dynastiewechsel im Perserreich nach dem Erlöschen von Kyros' männlicher Linie. ${ }^{19}$ De facto war er ein Usurpator, weil sich die Tradition, auf die er sich berief, nicht auf das Reich bezog, das er 522/21 v. Chr. übernahm, sondern auf ein politisches Gebilde der Vergangenheit, das durch Kyros' Eroberungen und die Entstehung eines neuen Herrschaftsbereichs obsolet geworden war.

\section{Naturkatastrophen als Omen für innerdynastische Konflikte}

Auch im Bereich des Hellenismus findet sich das literarische Motiv der Verbindung von Naturkatastrophen mit Brüchen von Herrschaftslinien. Ein anschauliches Beispiel bieten die Quellenberichte zur gescheiterten Dynastiebildung des Lysimachos. Er war einer der Generäle Alexanders des Großen, die nach dessen frühem Tod 323 v. Chr. in Babylon sein Riesenreich unter sich aufgeteilt, als seine Nachfolger - griechisch diadochoi - eigene Reiche gegründet und insgesamt sechs Kriege um ihren territorialen Bestand geführt hatten (Heinen 2003, 32f.).

Während sich die Dynastien der Ptolemäer in Ägypten, der Seleukiden in Teilen Syriens, der Antigoniden in Makedonien und etwas später der Attaliden in Pergamon etab-

18 Anšan wurde ein Teil des Reichsgebiets. Zu Anšan vgl. Rollinger, 2006, 41.

19 Geht man davon aus, dass der gestürzte Gaumata tatsächlich der echte Bardiya gewesen ist, vollzog sich dieser zweite Machtwechsel in umgekehrter Richtung: von den Teispiden zu den Achaimeniden. 
lierten, gelang es Lysimachos nicht, sein Herrschaftsgebiet - Thrakien und Makedonien sicher an seine Nachfolger zu übergeben. Schuld waren Erbstreitigkeiten an seinem Hof. Lysimachos' ältester Sohn Agathokles war jahrelang als Thronfolger aufgebaut worden, hatte sich militärisch bewährt und bereits seine eigene Familie gegründet (Paus. 1,9,6; 1,10,3; Just. 17,1,4), als sein Vater im Zuge seiner Heiratspolitik zur Sicherung eines Bündnisses mit Ptolemaios von Ägypten dessen Tochter Arsinoë zur Frau genommen hatte (Paus. 1,10,3; Plut. Demetr. 31,5. Vgl. Carney 1994, 124; Lund 1992, 88). Aus dieser Ehe waren drei Söhne entstanden, die von Lysimachos zunehmend in den Vordergrund gerückt und gefördert worden waren, während Agathokles in wachsender Eigenständigkeit in Kleinasien militärisch operiert und anscheinend dabei nicht immer im Einklang mit der politischen Linie seines Vaters gehandelt hatte (Carney 1994, 126f.; Lund 1992, 186-198; Landucci Gattinoni 1992, 209-214; Grainger 1990, 178). Die Entfremdung zwischen Lysimachos und seinem Sohn endete mit Agathokles' Beseitigung 283/2 v. Chr. Die antike Überlieferung ist spärlich und höchst problematisch, da sie von misogynen Stereotypen und Tyrannentopik verzerrt ist. ${ }^{20}$ Als treibende Kraft gilt Arsinoë, die als eine intrigante, herrschsüchtige und skrupellose femme fatale geschildert wird. Dieses Klischee entspricht der generellen Reaktion von griechischen Autoren auf Königinnen, die auf dem politischen Parkett Präsenz zeigten. Der Handlungsraum, der sich ihnen als Mitgliedern eines Herrscherhauses in monarchischen Strukturen eröffnete, war den griechischen Verfassern, die in stadtstaatlichen Strukturen sozialisiert worden waren, ein Rätsel (Carney 1996, 566). Auf der anderen Seite trägt Lysimachos' Porträt die Züge des Tyrannen: ein grausamer, ungerechter Willkürherrscher, der sich von seinen Trieben lenken lässt und daher den Einflüsterungen seiner jungen Frau gehorcht (App. Syr. 64; Just. 17,1,1-5; Lukian, Ikaromenipp. 15; Strab. 13,4,1). „Es pflegt für den Menschen viel Unheil aus der Liebe zu erwachsen“, beginnt Pausanias entsprechend seinen Bericht $(1,10,3) .{ }^{21}$ Als eine wichtige Quelle der erhaltenen, indes späteren Zeugnisse zum Untergang von Lysimachos' Haus gilt die Diadochengeschichte des Hieronymos von Kardia, in die Inhalte von Seleukos' Propaganda eingeflossen sein sollen (Lund 1992, 193; Hadley 1969, 141-145). ${ }^{22}$ Pausanias verweist zudem auf Hieronymos' ohnehin feindselige Haltung gegenüber Lysimachos, die den Grundtenor zusätzlich verschärft haben wird: Er habe ihn gehasst, weil er seine Heimatstadt Kardia zerstört hatte (Paus. 1,9,8), um seine Residenz Lysimacheia zu gründen (App. Syr. 1,1) und Kardias Bewohner dort anzusiedeln.

Die Krise an Lysimachos' Hof wurde von seinem Gegner Seleukos genutzt, der Kriegshandlungen gegen ihn aufnahm, in deren Verlauf Lysimachos den Tod fand (Just. 17,1,7-8; App. Syr. 62). Die Nachfolge war ungesichert geblieben, die Dynastiebildung

20 Literarische Hauptquellen für die geschilderten Ereignisse sind: Justin, der die verlorenen Historia Philippicae des Pompeius Trogus aus dem 1. Jh. v. Chr. exzerpierte, und aktuell überwiegend um $400 \mathrm{n}$. Chr. datiert wird (zu seinen Quellen vgl. Forni/Angeli Bertinelli 1982); Appian aus Alexandria, der um die Mitte des 2. Jh. n. Chr. eine Geschichte Roms schrieb (vgl. Brodersen 1993; zu Appians Quellen über Seleukos' Krieg gegen Lysimachos vgl. Brodersen 1989, 188f.), und Pausanias, der im dritten Viertel des 2. Jh. n. Chr. eine Beschreibung Griechenlands verfasste und darin wenig Auskunft über seine Quellen gibt (vgl. Swain 1996, 330-356; Ameling 1996).

21 Eigene Übersetzung.

$22 \mathrm{Zu}$ Hieronymos von Kardia, der von 316-272 v. Chr. am antigonidischen Hof lebte, vgl. Schmitt $2005^{3}$, 456; Hadley 1969, 144. 
gescheitert. Makedonien und Thrakien wurden zum Zankapfel verschiedener Konkurrenten, bis sich die Antigoniden durchsetzten.

In der literarischen Tradition geht dem Nachfolgekonflikt eine Naturkatastrophe voran, die in den Quellen als Vorankündigung des Unglücks interpretiert wird: „Etwa zu derselben Zeit war in den Gegenden des Hellespont und der Chersones ein Erdbeben, wobei vor allem die Stadt Lysimacheia, zweiundzwanzig Jahre zuvor vom König Lysimachos gegründet, zerstört wurde. Dieses schlechte Vorzeichen verkündete Lysimachos und seiner Familie etwas Schreckliches, dem Königtum den Fall samt schweren Schaden der heimgesuchten Landstriche. Und der Prophezeiung fehlte nicht die Bestätigung, denn nach kurzer Zeit brachte er seinen Sohn Agathokles, den er schon für die Nachfolge der Regierung bestimmt hatte (...), in einer Anwandlung des Hasses (...) und unter Beihilfe der Stiefmutter Arsinoë durch Gift ums Leben“ (Just. 17,2,1-5). ${ }^{23}$ Elemente der seleukidischen Propaganda, wonach Seleukos den Krieg - de facto expansiver Natur (Just. 17,1,11; Nep. de reg. 3,2-3) - geführt hatte, um Agathokles zu rächen, scheinen hier durchzuschimmern. Da Seleukos nach Lysimachos' Tod dessen überlebende Söhne verdrängte und sich zum makedonischen König ausrufen ließ (Paus. 1,16,1; Just. 17,2,4), musste er den Bruch der Herrschaftskontinuität neutralisieren, um die Akzeptanz der Makedonen zu gewinnen. Abermals dient das literarische Stilmittel der Katastrophenbeschreibung dazu, eine legitimierende Tradition zu kreieren. Seleukos stellte sich als Heilsbringer dar, der Makedonien von Lysimachos und seiner zur Tyrannei entarteten Herrschaft befreit hatte. Lysimachos' Wandel zum Unheilskönig, seine topische Negativentwicklung vom aufrechten Philosophenschüler, der gegen einen Gewaltherrscher opponiert (Just. 15,3), zum Tyrannen, der nicht minder willkürlich handelt (Just. 17,1,4), wurde durch das Erdbeben als warnendes Zeichen der Götter unterstrichen. ${ }^{24}$ Ausgerechnet seine Residenzstadt Lysimacheia mit dem politischen Zentrum des Hofs wurde zerstört, in straftheologischer Deutung eine Präfiguration des Untergangs seines Königshauses, das Scheitern der Dynastiebildung.

\section{Fazit}

Es wurde aufgezeigt, dass zwischen den verschiedenen Rollen der Götter und Akteure im Kontext antiker Deutungsmuster von Naturkatastrophen und -phänomenen stärker zu differenzieren ist. Die Götter treten als Warnende und Bestrafende auf, als Helfer, wenn die Unheilsszenarien als „Katastrophe der Rettung“ und „Katastrophe der anderen“ (Kehne 1998, 374) interpretiert werden - dies hängt jeweils von der Perspektive der Berichterstattung ab - oder sie ziehen sich ganz zurück und bewirken mit ihrer Absenz ein Kippen der Naturvorgänge. Die negativ geschilderten Akteure führen die Naturerscheinungen entweder unbewusst oder bewusst durch ihr Verhalten herbei. Positiv konnotierte Akteure werden meist als Göttergünstlinge dargestellt, die dazu bestimmt und befähigt sind, die Katastrophe zu bewältigen und die Krise zu beenden. Gewarnte oder bestrafte Akteure

23 Zitiert nach der Übersetzung von O. Seel.

24 Da Apollon, der in der griechischen Tradition auch als Urheber von Erdbeben auftreten konnte (Bredow 1998, 165) seit Seleukos' Nachfolger Antiochos I. als göttlicher Stammvater der Seleukiden galt (Just. 15,4,3-6; App. Syr. 56. Vgl. LeRider/Callataÿ 2006, 45-49; Mehl 1986, 6-12), ist es auch möglich, dass sich diese Deutung erst in dieser Zeit entwickelte. Relativiert wurde Lysimachos' Negativporträt etwa durch Landucci Gattinoni 1992; Lund 1992; Heckel 1982, 374-377. 
wie Lysimachos leiden unter der Katastrophe und ihren Folgen; als eine Steigerung der Negativschilderung ist das Motiv zu betrachten, wonach die Akteure das Chaos als ihr ureigenes Element betrachten. Da in der Antike die Deutung von Naturkatastrophen als verschieden gearteter - Ausdruck des Götterwillens vorherrschte und den Lauf der Natur untrennbar mit der göttlichen Ordnung verknüpfte, lag als Konsequenz nah, mit Katastrophenszenarien Fragen der Legitimität eines Herrschers, dem die Position des irdischen Bewahrers dieser Ordnung zukam, zu verbinden. In den ägyptischen Schilderungen von Unheilsregimes gilt das Umkippen der Natur als Beleg für die Unrechtmäßigkeit von Herrschaft. Auch Dareios I. folgt diesem Modell, wenn er in der Behistun-Inschrift verkündet, erst durch seine Intervention gegen Gaumata und seine Thronbesteigung die göttliche Ordnung wieder etabliert zu haben.

Besonders häufig treten daher Katastrophenszenarien in politischen Diskursen im Zusammenhang mit Brüchen der Herrschaftskontinuität auf: in den thematisierten Beispielen bei der persischen respektive makedonischen Eroberung Ägyptens, Dareios' Machtergreifung im Perserreich und das Erlöschen von Lysimachos' Haus - oder Brüchen von politischen Traditionen - im Fall von Lysimachos ist als weiterer Faktor sein Wandel zum Tyrannen zu nennen. Allgemein gesprochen wurde das literarische Motiv des Herrschers, dessen Regierung von Naturkatastrophen begleitet wurde, offenbar als ein adäquates Mittel betrachtet, einen Amtsvorgänger abzuwerten. In Situationen des politischen Wandels sollten die Kontinuitätsbrüche durch die Berufung darauf neutralisiert werden, dass an die göttlich vorgegebene, einst in mythischer Vorzeit etablierte Herrschaftstradition angeknüpft wurde. Dieser Vorstellung gemäß wurde das Gewandelte rechtfertigend als eine Rückkehr zu den eigentlichen Wurzeln dargestellt.

\section{Bibliographie}

Ameling, W. (1996) Pausanias und hellenistische Geschichte. In: Bingen, J. (Hg.), Pausanias historien. Genf: Fondation Hardt: 117-160.

Assmann, J. (2004) Ägyptische Geheimnisse. München: Wilhelm Fink.

Assmann, J. (1991) Stein und Zeit. Mensch und Gesellschaft im Alten Ägypten. München: Fink.

Baines, J. (1996) On the composition and inscriptions of the Vatican statue of Udjahorresne. In: Der Manuelian, P. (Hg.), Studies in honor of W.K. Simpson, I. Boston: Department of Ancient Egyptian, Nubian, and Near Eastern, Museum of Fine Arts: 8393.

Balcer, J. M. (1987) Herodotus and Bisitun. Problems in ancient Persian historiography. Stuttgart: Steiner.

Binder, G. (1964) Die Aussetzung des Königskindes Kyros und Romulus. Meisenheim am Glan: Hain.

Blasius, A./Schipper, B.U. (2002) Apokalyptik und Ägypten? - Erkenntnisse und Perspektiven. In: Dies. (Hg.), Ägyptische Apokalyptik. Eine kritische Analyse der relevanten Texte aus dem griechisch-römischen Ägypten. Leuven/Paris: Peeters: 277-302.

Bredow, I. v. (1998) Die mythischen Bilder der Naturkatastrophen. In: Olshausen, E./Sonnabend, H. (Hg.), Naturkatastrophen in der antiken Welt. Stuttgarter Kolloquium zur historischen Geographie des Altertums 6. Stuttgart: Steiner: 162-168.

Bresciani, E. (19963) The Persian occupation of Egypt. In: The Cambridge History of Iran II: $502-528$. 
Bresciani, E. (1998) „Il pleut sur la pierre“. Prophéties politiques dans la litterature démotiques. In: Assmann, J./Blumenthal, E. (Hg.), Literatur und Politik im pharaonischen und ptolemäischen Ägypten. Kairo: Institut francais d'archéologie orientale: 279-284.

Briant, P. (1996) Histoire de l'empire perse de Cyrus à Alexandre. Paris: Fayard.

Briant, P. (2002) History and ideology: The Greeks and „Persian decadence“. In: Harrison, T. (Hg.), Greeks and barbarians. Edinburgh: Edinburgh University Press: 193336.

Brodersen, K. (Hg.) (1989) Appians Abriss der Seleukidengeschichte (Syriake 45,23270,369). Text und Kommentar. München: Editio Maris.

Brodersen, K. (1993) Appian und sein Werk. In: Aufstieg und Niedergang der Römischen Welt II 34.1: 339-363.

Brosius, M. (1998) Women in ancient Persia (559-331 B.C.). Oxford: Oxford University Press.

Brown, T.S. (1982) Herodotus' portrait of Cambyses. In: Historia 31: 387-403.

Carney, E.D. (1994): Arsinoe before she was Philadelphus. In: The Ancient History Bulletin 8: 123-131.

Carney, E.D. (1996) Alexander and Persian women. In: American Journal of Philology 117: 563-583.

Clauss, M. (2001) Das Alte Ägypten. Berlin: Alexander Fest Verlag.

Cook, J.M. $\left(1996^{3}\right)$ The rise of the Achaemenids and the establishment of their empires. In: The Cambridge History of Iran II: 200-291.

Cool Root, M. (1979) King and kingship in Achaemenid art. Essays on the creation of iconography of empire. Leiden: Brill.

Demandt, A. (1996) Darius und der „falsche“ Smerdis 522 v. Chr. In: Ders. (Hg.), Das Attentat in der Geschichte. Köln: Böhlau: 9-25.

Depuydt, L. (1995) Murder in Memphis: The story of Cambyses's mortal wounding of the Apis bull (ca. 523 B.C.E.). In: Journal of Near Eastern Studies 54: 119-126.

Desmond, W. (2004) Punishments and the conclusion of Herodotus' Histories. In: Greek, Roman and Byzantine Studies 44: 19-40.

Dillery, J. (2005) Cambyses and the Egyptian Chaosbeschreibung tradition. In: Classical Quarterly 55: 387-406.

Evans, J.A.S. (1965) Despotes nomos. In: Athenaeum 43: 142-153.

Fornara, C.W. (1990) Human history and the constraint of fate in Herodotus. In: Allison, J.W. (Hg.), Conflict, antithesis, and the ancient historian. Columbus: Ohio State University Press: 25-45.

Forni, G./Angeli Bertinelli, M.G. (1982) Pompei Trogo come fonte di storia. In: Aufstieg und Niedergang der Römischen Welt II 30.3: 1298-1362.

Funck, B. (1996) „König Perserfreund“. Die Seleukiden in der Sicht ihrer Nachbarn. In: Ders. (Hg.), Hellenismus. Beiträge zur Erforschung von Akkulturation und politischer Ordnung in den Staaten des hellenistischen Zeitalters. Tübingen: Mohr: 195-215.

Gammie, J.G. (1986) Herodotus on kings and tyrants: Objective historiography or conventional portraiture. In: Journal of Near Eastern Studies 45: 171-195.

Grainger, J. D. (1991) Hellenistic Phoenicia. Oxford: Clarendon Press.

Groh, D./Kempe, M./Mauelshagen, F. (2003) Einleitung. Naturkatastrophen - wahrgenommen, gedeutet, dargestellt. In: Dies. (Hg.), Naturkatastrophen: Beiträge zu ihrer Wahrnehmung, Deutung und Darstellung in Text und Bild von der Antike bis ins 20. Jahrhundert. Stuttgart: Narr: 11-33.

Hadley, R.A. (1969) Hieronymus of Cardia and early Seleucid mythology. In: Historia 18: 142-152. 
Harrison, T. (2003) Upside down and back to front: Herodotus and the Greek encounter with Egypt. In: Matthews, R./Roemer, C. (Hg.), Ancient perspectives on Egypt. London: UCL Press: 145-155.

Heckel, W. (1982) The early career of Lysimachos. In: Klio 64: 373-381.

Heinen, H. (2003) Geschichte des Hellenismus von Alexander bis Kleopatra. München: Beck.

Heinrichs, J. (2001) Rezension zu R. Bichler, Herodots Welt, Berlin 2000. In: Göttinger Forum für Altertumswissenschaft 4: 1169-1171.

Heinrichs, J. (1989) Ionien nach Salamis. Die kleinasiatischen Griechen in der Politik und politischen Reflexion des Mutterlandes. Bonn: Habelt.

Hölbl, G. (1994) Geschichte des Ptolemäerreiches. Politik, Ideologie und religiöse Kultur von Alexander bis zur römischen Eroberung. Darmstadt: Wissenschaftliche Buchgesellschaft.

Hoffmann, F. (2000) Ägypten. Kultur und Lebenswelt in griechisch-römischer Zeit. Eine Darstellung nach den demotischen Quellen. Berlin: Akademie Verlag.

Hopfner, T. (1967) Plutarch. Über Isis und Osiris. Text, Übersetzung und Kommentar, I. Darmstadt: Wissenschaftliche Buchgesellschaft.

Huß, W. (1997) Ägyptische Kollaborateure in persischer Zeit. In: Tyche 12: 131-143.

Kehne, P. (1998) Ein Altar für die Winde. Die persische Flottenkatastrophen 480 v. Chr. In: Olshausen, E./Sonnabend, H. (Hg.), Naturkatastrophen in der antiken Welt. Stuttgarter Kolloquium zur historischen Geographie des Altertums 6. Stuttgart: Steiner: 364-375.

Kent, R.G. (1946) The oldest Old Persian inscription. In: Journal of the American Oriental Society 66: 202-212.

Kuhrt, A. (1995) The Ancient Near East c. 3000-330 B.C., I-II. London: Routledge.

Kuhrt, A. (2003) Making history: Sargon of Agad and Cyrus the Great of Persia. In: Achaemenid History 13: 347-361.

Landucci Gattinoni, F. (1992) Lisimaco di Tracia. Un soprano nella prospettiva del primo ellenismo. Mailand: Editoriale Jaca.

Lateiner, D. (1977) No laughing matter: A literary tactic in Herodotus. In: Transactions of the American Philological Association 107: 173-182.

Lateiner, D. (1989) The historical method of Herodotus. Toronto: University of Toronto Press.

LeRider, G./Callataÿ, F. de (2006) Les Séleucides et les Ptolémées. L'héritage monétaire et financier d'Alexandre le Grand. Paris: Éd. du Rocher.

Lloyd, A.B. (2002) Egypt. In: Bakker, E.J. u.a. (Hg.), Brill's companion to Herodotus. Leiden: Brill: 415-435.

Lloyd, A.B. (1990) Herodotus on Egyptians and Libyans. In: Nenci, G./Reverdin, O. (Hg.), Hérodote et les peuples non grecs, Genf: Fondation Hardt: 215-244.

Lund, H.S. (1992) Lysimachus. A study in early Hellenistic kingship. London: Routledge.

Marcuse, L. (1985) Die Welt der Tragödie. Frankfurt a.M.: Fischer.

Mehl, A. (1986) Seleukos Nikator und sein Reich. Leuven: Peeters.

Moyer, I.S. (2002) Herodotus and an Egyptian mirage: the genealogies of the Theban priests. In: Journal of Hellenic Studies 122: 70-90.

Müller, S. (2007) Luxus, Sittenverfall, Verweichlichung und Kriegsuntüchtigkeit: Die Codes der Dekadenz in den antiken Quellen. In: Hoffstadt, C. (Hg.), Dekadenzen. Bochum/Freiburg: Projekt Verlag: 13-52. 
Müller, S. (2008) Das antike Persien im Ausnahmezustand. Dareios I. im Kampf gegen die „Lüge“. In: Ruf, O. (Hg.), Ästhetik der Ausschließung. Würzburg: Königshausen $\&$ Neumann (i.D.).

Murray, O. (2001) Herodotus and oral history; Herodotus oral history reconsidered. In: Luraghi, N. (Hg.), The historian's craft in the age of Herodotus. Oxford: Oxford University Press: 16-44, 314-325.

Nagel, W. (1972/75) Herrscher in der Bildkunst, § 9.3.1. In: Reallexikon der Assyriologie und Vorderasiatischen Archäologie, 4: 356-361.

Munson, R.V. (1991) The madness of Cambyses (Herodotus 3,16-38). In: Arethusa 24: 43-65.

Mylonopoulos, J. (1998) Poseidon, der Erderschütterer. Religiöse Interpretationen von Erd- und Seebeben. In: Olshausen, E./Sonnabend, H. (Hg.), Naturkatastrophen in der antiken Welt. Stuttgarter Kolloquium zur historischen Geographie des Altertums 6. Stuttgart: Steiner: 82-89.

Posener, G. (1936) La première domination Perse en Égypte. Recueil d'inscriptions hiéroglyphiques. Kairo: Institut francais d'archéologie orientale.

Raaflaub, K. (1985) Die Entdeckung der Freiheit. Zur historischen Semantik und Gesellschaftsgeschichte eines politischen Grundbegriffes der Griechen. München: Beck.

Rollinger, R. (2006) Ein besonderes historisches Problem. Die Thronbesteigung des Dareios und die Frage seiner Legitimität. In: Historisches Museum der Pfalz Speyer (Hg.), Pracht und Prunk der Großkönige. Das persische Weltreich. Stuttgart: Theiss: 41-53.

Sancisi-Weerdenburg, H. (2005) The personality of Xerxes, king of kings. In: Bakker, E.J. u.a. (Hg.), Brill's Companion to Herodotus. Leiden: Brill: 579-590.

Schmitt, H.H. (2005³) Hieronymos. In: Ders./Vogt, E. (Hg.), Lexikon des Hellenismus. Wiesbaden: Harrassowitz: 456-457.

Serrano Delgado, J.M. (2004) Cambyses in Sais. In: Chronique d' Égypte 79: 31-52.

Sonnabend, H. (1999) Naturkatastrophen in der Antike: Wahrnehmung, Deutung, Management. Stuttgart: Metzler.

Sonnabend, H. (1998) Hybris und Katastrophe. Der Gewaltherrscher und die Natur. In: Olshausen, E./Sonnabend, H. (Hg.), Naturkatastrophen in der antiken Welt. Stuttgarter Kolloquium zur historischen Geographie des Altertums 6. Stuttgart: Steiner: 34-41.

Sonnabend, H. (2003) Wahrnehmung von Naturkatastrophen in der Antike: Das Kampanien-Erdbeben von 62 n. Chr. und der Ausbruch des Vesuv 79 n. Chr. In: Groh, D. u.a. (Hg.), Naturkatastrophen: Beiträge zu ihrer Wahrnehmung, Deutung und Darstellung in Text und Bild von der Antike bis ins 20. Jahrhundert. Stuttgart: Narr: 37-44.

Stephens, S.A. (2003) Seeing double: Intercultural poetics in Ptolemaic Alexandria. Berkeley/London: University of California Press.

Swain, S. (1996) Hellenism and empire. Language, classicism, and power in the Greek world $A D$ 50-250. Oxford: Clarendon.

Thissen, H.-J. (2002) Das Lamm des Bokchoris. In: Blasius, A./Schipper, B.U. (Hg.), Ägyptische Apokalyptik. Eine kritische Analyse der relevanten Texte aus dem griechisch-römischen Ägypten. Leuven/Paris: Peeters: 113-138.

Tuplin, C. (1991) Darius' Suez canal and Persian imperialism. In: Achaemenid History 6: 237-283.

Turner, E.G. (1984²) Ptolemaic Egypt. In: The Cambridge Ancient History VII, 1: 118174. 
Vittmann, G. (2003) Ägypten und die Fremden im ersten vorchristlichen Jahrtausend. Mainz: von Zabern.

Waldherr, G. (1998) Altertumswissenschaften und moderne Katastrophenforschung. In: Olshausen, E./Sonnabend, H. (Hg.), Naturkatastrophen in der antiken Welt. Stuttgarter Kolloquium zur historischen Geographie des Altertums 6. Stuttgart: Steiner: 51-64.

Wiesehöfer, J. (2005³) Das antike Persien von 550 v. Chr. bis 650 n. Chr. Düsseldorf: Albatros.

Wildung, D. (1977) Geschichtsdarstellung. In: Lexikon der Ägyptologie 2: 564-566.

Zimmermann, B. (1986) Die griechische Tragödie. Eine Einführung. München/Zürich: Artemis.

Sabine Müller ist Privatdozentin für Alte Geschichte am Historischen Seminar der Leibniz-Universität Hannover. Ihre Forschungsschwerpunkte sind das Achaimenidenreich, das argeadische Makedonien und der Hellenismus, insbesondere die mediale Inszenierung des ptolemäischen Herrscherpaars, antike Fremdenbilder sowie Antikenrezeption in europäischer Kunst.

E-Mail: sabine.mueller@hist.uni-hannover.de 